\title{
Réalisation d'une cellule à cisaillement elliptique
}

\author{
B. Noetinger, L. Petit, E. Guazzelli (*) et M. Clément \\ Laboratoire d'Hydrodynamique et de Mécanique Physique, Unité associée au C.N.R.S. n 857, E.S.P.C.I., \\ 10, rue Vauquelin, 75005 Paris, France
}

(Reçu le 23 juin 1986, révisé le 12 janvier 1987, accepté le 5 juin 1987)

\begin{abstract}
Résumé. - Nous décrivons un système expérimental permettant de soumettre un fluide à un mouvement de cisaillement alternatif (fréquence de 50 à $300 \mathrm{~Hz}$, amplitude de déplacements de 2 à $20 \mu \mathrm{m}$ ) polarisé elliptiquement, circulairement ou rectilignement (taux de cisaillement variant d'environ 10 à $1000 \mathrm{~s}^{-1}$ ) et de réaliser dans le même temps des observations optiques (microscopie, diffraction) des structures induites par le cisaillement ; nous pouvons également évaluer la viscosité du fluide dans ces conditions d'écoulement dans une gamme allant de quelques dizaines à quelques centaines de poises. Après avoir été développé pour étudier des instabilités convectives dans les cristaux liquides, ce système est actuellement utilisé pour observer des mises en structures de suspensions et évaluer leur viscosité.
\end{abstract}

\begin{abstract}
We describe an experimental device which allows us to subject a fluid to an alternating shear motion (frequency 50 to $300 \mathrm{~Hz}$, amplitude of displacement 2 to $20 \mu \mathrm{m}$ ) elliptically, circularly or linearly polarized (shear rate 10 to $1000 \mathrm{~s}^{-1}$ ) and simultaneously, to perform optical observations (microscopy, diffraction) of the ordering induced by the shear, as well as viscosity estimation in the range few tens to few hundred poises. After having been developed to study convective instabilities in nematic liquid crystals, this set-up is currently used to study the ordering of sheared suspensions, and their viscosity.
\end{abstract}

\section{Introduction.}

Nous décrivons dans cet article un ensemble expérimental permettant de produire un mouvement de cisaillement alternatif dans un fluide disposé entre deux plans parallèles, et d'étudier le fluide sous microscope et par diffraction laser. Ce système a été développé dans notre laboratoire à partir de l'année 1982 pour étudier les instabilités d'un cristal liquide nématique soumis à un mouvement de cisaillement alternatif polarisé elliptiquement; nous avons pu ainsi étudier la géométrie des structures convectives induites par le cisaillement [1]; notre propos dans cet article est de décrire l'ensemble du dispositif expérimental, en particulier le système original permettant de créer le mouvement de cisaillement elliptique à l'intérieur du fluide.

L'intérêt de ce système est de pouvoir appliquer au fluide deux mouvements de cisaillement dans deux directions perpendiculaires, le mouvement

(*) Département de Physique des Systèmes Désordonnés, Université de Provence, Centre Saint Jérome, 13397 Marseille Cedex 13, France. dans l'une des directions pouvant être considéré comme une perturbation du mouvement dans l'autre direction; de plus, la théorie de la réponse d'un milieu anisotrope (cristal liquide) à ce type de mouvement polarisé elliptiquement a été faite [2] et un certain nombre de résultats expérimentaux ont été obtenus dans ces conditions $[3,4]$; d'autre part, l'utilisation de mouvements alternatifs permet de s'affranchir d'un effet d'entraînement du fluide par l'écoulement moyen (comme dans le cas du « rhéomètre à bille » précédemment développé dans notre laboratoire [5]). Enfin, les quantités de produit nécessaires sont très petites (une fraction de $\mathrm{cm}^{3}$ ).

Récemment, nous avons utilisé ce même dispositif pour étudier la mise en ordre de suspension soumises à un mouvement de cisaillement elliptique, et évaluer leur viscosité. Nous décrivons les modifications apportées au système pour réaliser ces mesures qui sont actuellement en cours [6].

\section{Description du système expérimental.}

2.1 La Cellule de Cisaillement. - Le dispositif de cisaillement et une vue en coupe détaillée sont représentés sur les figures $1 \mathrm{a}$ et $1 \mathrm{~b}$. 

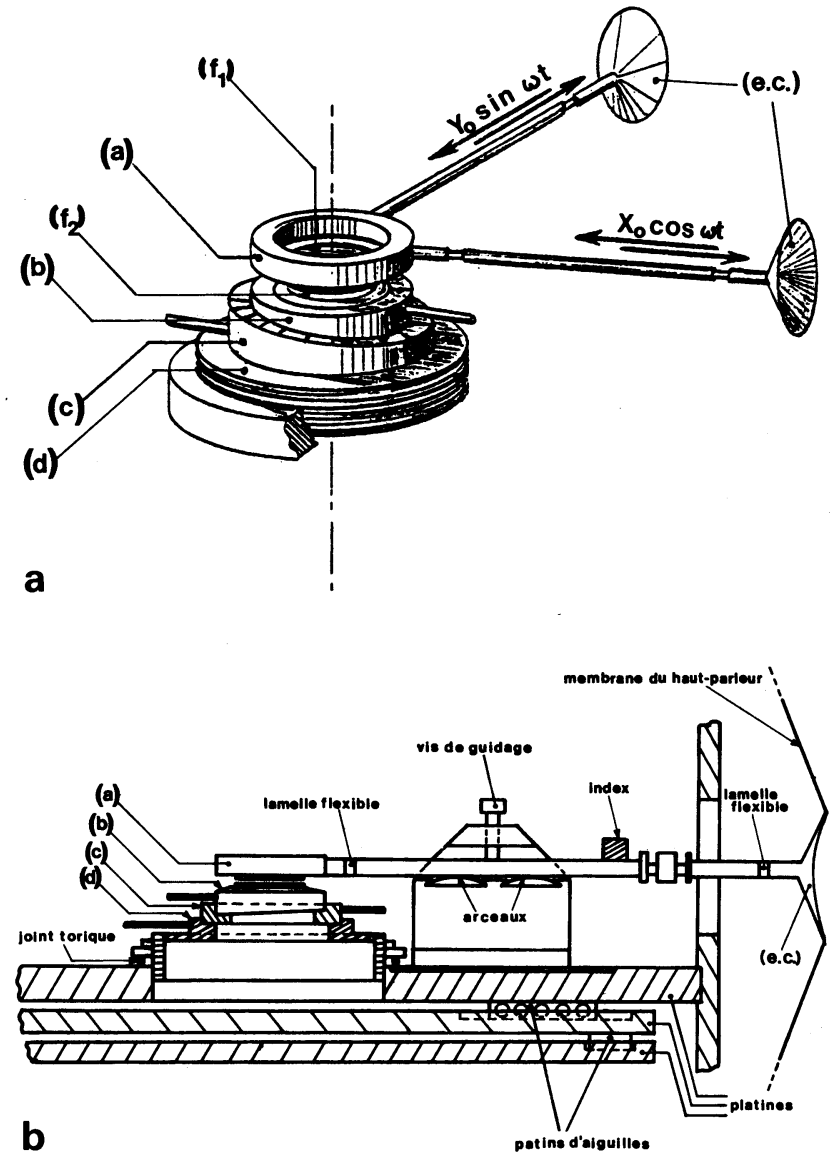

Fig. 1. - Dispositif expérimental pour produire le mouvement de cisaillement - $a$ : la cellule de cisaillement - $b$ : vue en coupe détaillée. $\left(f_{1}\right)$ : fenêtre supérieure mobile (disque en verre), (a): porte-fenêtre supérieur, $\left(f_{2}\right)$ : fenêtre inférieure fixe, (b) : porte-fenêtre inférieur, (c) : bague, (d) bague filetée, (e.c.) : embout conique.

[Experimental set-up to generate the shear motion - a : the shear cell - $b$ : cross-section. $\left(f_{1}\right)$ : top-window, movable (circular glass plate); (a) : top-window bearing; $\left(f_{2}\right)$ : lower window, fixed; (b) : lower window bearing ; (c) : ring; (d) : threaded ring; (e.c.) : conical adapter (for loudspeaker connecting).]

Deux disques épaulés en verre $\left(\mathrm{f}_{1}\right.$ et $\left.\mathrm{f}_{2}\right)$ de diamètre $15 \mathrm{~mm}$, d'épaisseur $2,5 \mathrm{~mm}$, polis à $N 10$, sont utilisés comme fenêtres; ils sont tenus en position sur les portes-fenêtres (a) et (b) par deux joints toriques; ce mode de fixation rend possible le démontage des fenêtres pour leur nettoyage.

Le porte-fenêtre supérieur (a) est fixé à deux bras perpendiculaires. Chacun d'eux est collé au centre de la membrane d'un haut-parleur par l'intermédiaire d'un embout conique évidé ; on peut ainsi communiquer au porte-fenêtre deux composantes de mouvement indépendantes perpendiculaires.

Chaque bras, de longueur réglable, comporte une tige rigide de très faible masse associée à un intercalaire fileté gauche-droite et repose sur deux lames recourbées en arceaux.
Une vis verticale en téflon située entre les deux arceaux maintient le déplacement du bras dans la direction horizontale; une autre vis, horizontale, fait office de butée latérale.

L'ensemble (porte-fenêtre, bras) présente quatre points d'appui et deux vis de maintien, en sus des fixations aux deux haut-parleurs ; il est donc hyperstatique ; cet état participe à une meilleure tenue du plan de déplacement du porte-fenêtre.

Afin de pallier la raideur des bras (qui pourrait être gênante dans le cas de déplacements importants), une ou deux «charnières » (lamelles de bronze au bérilium) ont été disposées verticalement sur chacun d'eux et autorisent un déplacement du porte-fenêtre dans le plan horizontal $X$ - $Y$.

Un index fixé sur chaque tige permet la mesure de l'amplitude et de la phase du déplacement de la plaque dans chaque direction : pour cela, on forme l'image de l'index éclairé par une fibre optique, sur une photodiode (UDT PIN 10 DP) à l'aide d'un objectif de microscope (PO X20 Olympus). On détecte ainsi la variation de l'intensité lumineuse due au mouvement de l'index. La photodiode délivre un signal électrique de même fréquence que le mouvement de la plaque, en opposition de phase avec celui-ci, et d'amplitude proportionnelle à l'amplitude du déplacement des plaques (voir § 2.2).

Les deux haut-parleurs utilisés (Siare $12 \mathrm{CP}$ ) sont identiques ; ils ont été choisis d'une part pour la largeur de leur bande passante, d'autre part pour leur masse relativement modérée (environ $1 \mathrm{~kg}$ ) afin que le centre de gravité de l'ensemble de la cellule ne soit pas trop déporté vers l'extérieur. Nous avons remplacé la platine du microscope qui permettait un déplacement de la cellule dans les deux directions horizontales (système à crémaillère) par un ensemble de trois platines métalliques pouvant glisser les unes sur les autres par l'intermédiaire de patins à aiguilles (Fig. 1-b) ; ceci permet de déplacer avec beaucoup plus de facilité l'ensemble (cellule, haut-parleur) pour viser au microscope une zone précise de l'échantillon.

La fenêtre inférieure $\left(f_{2}\right)$ est solidaire d'un couple de bagues (b) et (c) (Fig. 1). Ces deux bagues sont coupées par un plan de même inclinaison $\left(\alpha=18^{\prime}\right)$. On peut ainsi produire un coin d'angle et de direction variable en faisant tourner les bagues l'une par rapport à l'autre (Fig. 2) (les angles ont été fortement exagérés sur la figure);

- partant de la position d'angle nul (Fig. 2-(1)), si on tourne la pièce supérieure (b) d'un demi-tour, on fait varier l'angle du coin jusqu'à sa valeur maximum : $2 \alpha=36$ ' (Fig. 2-(2)). Treize graduations gravées sur une demi-circonférence de la bague (c) sont destinées au repérage de l'angle du coin (pour la mesure de cet angle, voir au \$ 3.1.2); une graduation repère est gravée sur la bague (b) ; 
(1)

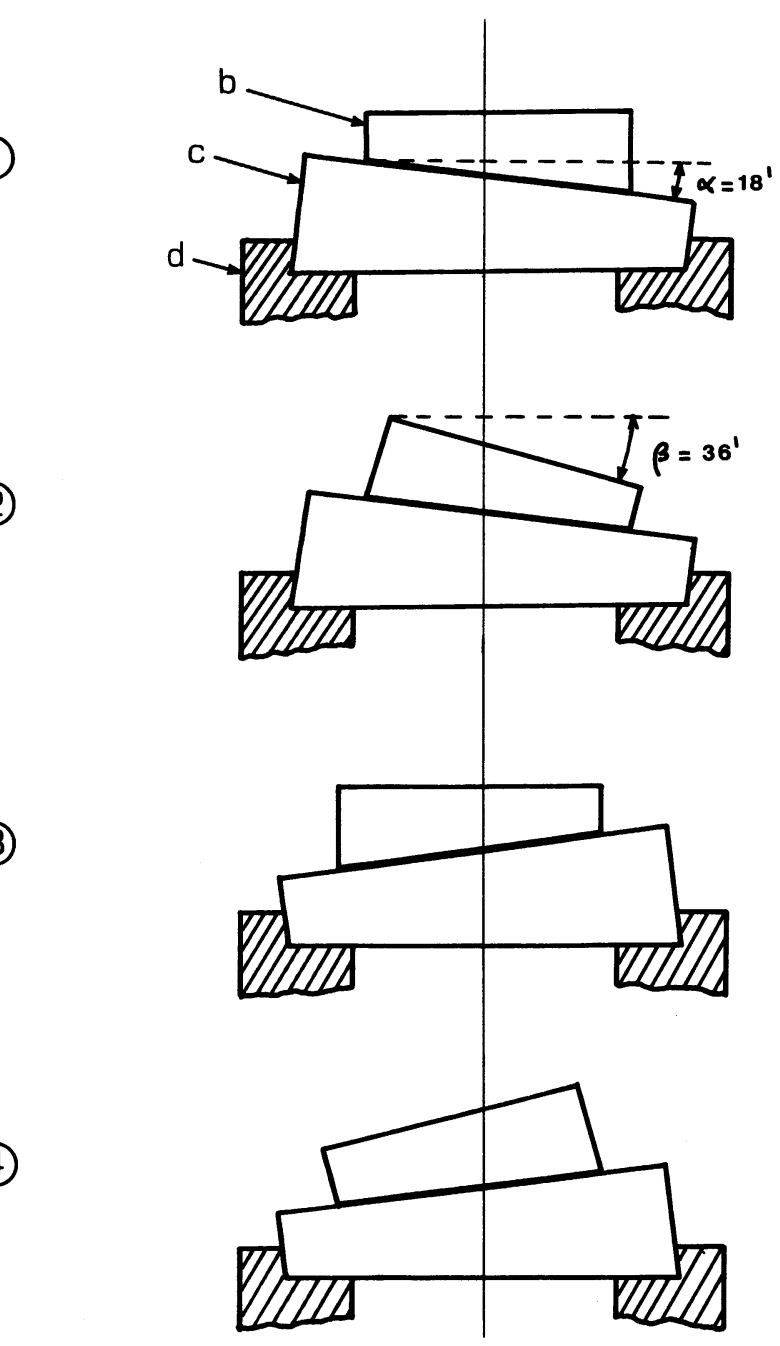

(2)

Fig. 2. - Les différentes positions du coin : (1) $\rightarrow$ (2) rotation d'un demi-tour de la bague (b) seule : variation de l'angle du coin de 0 à 36 . (2) $\rightarrow$ (4) rotation d'un demi-tour des bagues (b) et (c) ensembles : rotation d'un demi-tour de l'arête du coin (d'angle $36^{\prime}$ dans ce cas).

[The various adjustements to obtain desired wedge positions, (1) $\rightarrow$ (2) [to go from figure 2-(1) to 2-(2) halfturn rotation of the ring (b) only : increase of the angle of the wedge from 0 to $36^{\prime},(2) \rightarrow(4)$ half-turn rotation of the rings (b) and (c) together : no change of the angle of the wedge ( $36^{\prime}$ in this case), but half-turn rotation of the ridge of the wedge.]

- si on tourne les deux pièces (b) et (c) simultanément, il n'y a pas de variation de l'angle du coin, seule change la direction de son arête (Fig. 2-(4)).

L'ensemble des deux pièces (b) et (c) est posé sur une troisième bague (d), fileté au pas de $0,5 \mathrm{~mm}$; la rotation de l'ensemble [(b), (c), (d)] permet un changement continu de l'épaisseur de la cellule sans modification de l'inclinaison du plan de la lame supérieure. La circonférence de la bague (d) est gravée de 50 divisions pour le choix de l'épaisseur (10 $\mu \mathrm{m}$ par division) (pour la mesure de l'épaisseur voir en 3.1.1).
2.2 COMMANDE ET DÉTECTION DU MOUVEMENT DE LA PLAQUE. - Le système de commande et de détection du mouvement de la plaque supérieure de la cellule est schématisé sur la figure 3.

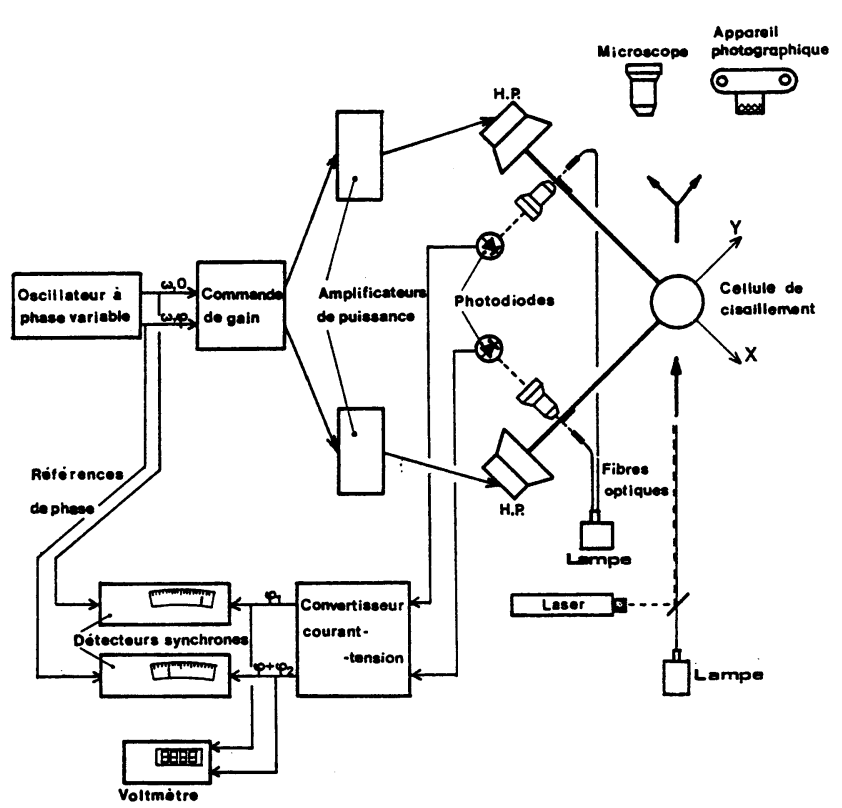

Fig. 3. - Schéma du système de commande et de détection du mouvement de la plaque.

[Schematic representation of the control and detection system.]

La chaîne de commande du mouvement comprend les éléments décrits ci-dessous :

- un oscillateur à phase variable (Feedback VPO 602) délivre deux signaux sinusoïdaux de même fréquence $f=\omega / 2 \pi$ et déphasés de $\varphi$ (ajustable);

- un système de commande de gain permet l'amplification simultanée des deux signaux, sans modification du rapport de leurs amplitudes, ni de leur déphasage; on peut ainsi maintenir constante l'ellipticité du mouvement. Ce système comporte trois modes de progression du gain :

- une cadence rapide, utile lorsqu'on désire faire progresser rapidement l'amplitude des déplacements de la plaque, jusqu'à des variations de $1 \mu \mathrm{m}$ toutes les $10 \mathrm{~s}$.

- une cadence lente, surtout utilisée dans les expériences sur les cristaux liquides quand on se rapproche de la zone d'instabilité à étudier ; on peut alors diminuer la progression de l'amplitude jusqu'à $1 \mathrm{~mm}$ toutes les $7 \mathrm{~min}$,

- enfin, une cadence manuelle pas à pas, qui permet d'encadrer au mieux les valeurs d'amplitude particulières (au voisinage d'un seuil d'instabilité par exemple) ;

- deux amplificateurs de puissance (Hewlett Packard 467 A) permettent d'alimenter les deux haut-parleurs (basse impédance : environ $7 \Omega$ à 
$200 \mathrm{~Hz}$ ) à courant sensiblement constant ; ceci est particulièrement important pour les déterminations de viscosité (voir au \$ 3.3).

La détection du mouvement de la plaque est assurée par le dispositif décrit au paragraphe 2.1 : fibre optique, index, objectif et photodiode :

- les photodiodes délivrent un courant, proportionnel au déplacement de la plaque dans chaque direction $X$ et $Y$; ces courants sont convertis en tension à l'aide d'un convertisseur à deux voies qui comporte d'une part un réglage de zéro (pour compenser le courant existant même en l'absence de mouvement) et d'autre part un réglage de gain (pour équilibrer les deux voies afin d'obtenir une même tension pour un même déplacement). Cependant, ce convertisseur n'est pas utilisé lors des mesures de viscosité, qui se font directement à partir de la phase du courant débité par les photodiodes. On évite ainsi d'introduire des déphasages supplémentaires dans la chaîne de mesure ;

- chaque signal est ensuite envoyé sur une détection synchrone (Brookdeal) dont la référence est fournie par l'oscillateur. Le déphasage $\varphi$ est alors ajusté de telle manière que les déplacements de la plaque dans les deux directions $X$ et $Y$ soient effectivement en quadrature; chaque point de la plaque décrit alors une ellipse d'axes $X$ et $Y$, dont l'excentricité peut être choisie à partir de l'amplitude des déplacements $X_{0}$ et $Y_{0}$;

- l'amplitude des signaux délivrés par les photodiodes est mesurée à l'aide d'un voltmètre numérique et permet la détermination des amplitudes de déplacement après un étalonnage grâce au microscope : la lame de verre est éclairée en stroboscopie à une fréquence voisine de celle du cisaillement ; l'amplitude de son déplacement est alors mesurée sur le réticule du microscope. L'incertitude sur l'étalonnage est de l'ordre de $1,5 \mu \mathrm{m}$.

2.3 RÉgulation thermiQue. - Un système de régulation thermique a été réalisé pour les expériences sur les cristaux liquides de façon à ce que les constantes d'élasticité et de viscosité de l'échantillon ne varient pas au cours d'une expérience. Le microscope et la cellule sont disposés dans une enceinte close à l'intérieur de laquelle circule un courant d'air dont la température est régulée par un ensemble $\{$ ventilateur, résistance chauffante, thermomètre à contact $\}$. On obtient ainsi une régulation à mieux que le degré, ce qui est suffisant pour nos expériences.

\section{Méthode expérimentale.}

3.1 ConTRÓle ET MESURE DES PARAMÈTRES GÉOMÉTRIQUES.

3.1.1 Epaisseur de l'échantillon. - Dans le cas des expériences sur les cristaux liquides nématiques qui sont des matériaux uniaxes optiques, l'épaisseur de l'échantillon est mesurée par interférométrie : la cellule est éclairée par un faisceau de lumière convergente, et, par suite de la biréfringence du cristal liquide on obtient une image conoscopique, constituée d'une croix noire au milieu de l'échantillon, et d'une série d'anneaux concentriques; la mesure du rayon du premier anneau permet de déterminer l'épaisseur de l'échantillon. Cette méthode n'est pas utilisable lorsqu'on travaille avec des suspensions, car ces milieux ne sont pas biréfringents et de plus très absorbants. Dans ce cas, nous mesurons l'épaisseur en visant la cellule par la tranche à l'aide du microscope dont un des objectifs (grossissement 7 fois) a est équipé d'un petit miroir très proche de la lentille frontale et incliné à $45^{\circ}$; par une mise au point sur le bord de la cellule, nous parvenons à mesurer la distance entre les lames de verre en utilisant le micromètre oculaire étalonné.

3.1.2 Angle du coin formé par les lames de verre. Un faisceau laser de rayons parallèles, étendu à la surface de la cellule à l'aide d'un montage afocal éclaire les deux lames de verre; les faisceaux réfléchis sur leur surface sont focalisés sur un écran grâce à une lentille de distance focale $f=610 \mathrm{~mm}$, donnant deux taches séparées d'une distance $\delta$; l'angle du coin vaut alors $\beta=\delta / 2 f$. Cette méthode offre une précision sur $\beta$ de l'ordre de $0,5 \mathrm{mrad}$, ce qui représente une variation d'épaisseur d'un bord à l'autre de la cellule d'environ $10 \mu \mathrm{m}$. Elle a été utilisée pour étalonner l'angle du coin en fonction des graduations portées sur l'anneau (c) de la cellule de cisaillement (Fig. 1 et paragraphe 2.1) ; l'étalonnage est reporté sur la figure 4.

Dans le cas des suspensions, le faisceau n'est pas élargi à cause des problèmes d'absorption, et on contrôle simplement le parallélisme des deux plaques en faisant coïncider les deux taches données à grande distance $(D \sim 5 \mathrm{~m})$ par réflexion du faisceau laser sur chacune des lames. Cette coïncidence est obtenue par action sur trois vis régulièrement disposées à la périphérie de l'anneau (a) posé lui-même sur un joint torique en caoutchouc (Fig. 1-b) (en effet, dans le cas de l'étude des suspensions qui sont beaucoup plus visqueuses que les cristaux liquides, nous avons été amenés à bloquer les rotations mutuelles des anneaux (b), (c) et (d) pour éviter une rotation de la lame inférieure sous l'effet du mouvement oscillant de la lame supérieure); par légère rotation des vis, on modifie progressivement l'angle du coin par écrasement du joint torique (environ $1 \mathrm{mrad}$ pour $1 / 8^{\circ}$ de tour de vis).

\subsection{OBSERVATION DES ÉCHANTILLONS.}

3.2.1 En microscopie. - Les structures convectives apparaissant dans les cristaux liquides, ainsi que la mise en ordre des suspensions sont observées à l'aide 


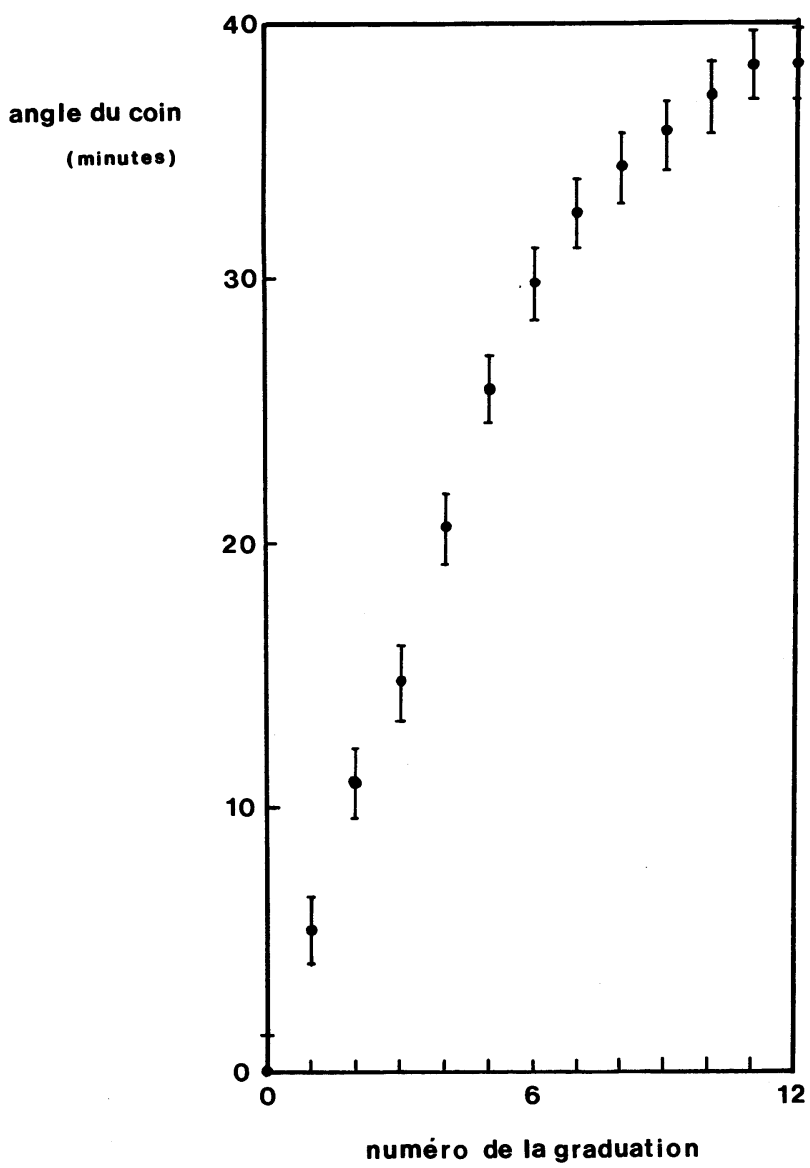

Fig. 4. - Etalonnage de l'angle du coin formé par les lames de verre.

[Calibration of the angle of the wedge between the glass plates.]

d'un microscope (Olympus Vanox) à des grossissements variant de 25 à 500 fois ; ce système d'observation est bien adapté à la taille des rouleaux convectifs dans les cristaux liquides et au diamètre des particules rigides dans les suspensions : quelques dizaines de microns dans les deux cas, ce qui est aussi l'ordre de grandeur de l'écartement entre les plaques.

Un dispositif de microphotographie permet de réaliser des clichés des observations effectuées.

Une caméra $16 \mathrm{~mm}$ permet de suivre l'évolution des structures convectives dans les cristaux liquides, l'arrangement géométrique des suspensions ou encore le mouvement des particules en suspension sous l'effet du cisaillement. Plus récemment, nous avons équipé le microscope d'une caméra vidéo à capteur CCD (Sony DXC 101P), qui grâce à sa masse très modérée $(550 \mathrm{~g})$ peut être montée directement sur le microscope ; ceci permet une étude beaucoup plus souple de la mise en structure des suspensions à partir d'enregistrements sur cassettes vidéo.

3.2.2 Par diffraction. - Un faisceau laser (hélium- néon $10 \mathrm{~mW}$ ) arrivant sous incidence normale sur la cellule permet d'étudier le réseau optique créé par les structures de l'échantillon (la période des structures est de quelques dizaines de microns).

3.3 DÉtermination DES Viscosités. - Pour avoir accès à la viscosité de l'échantillon, une première possibilité était de mesurer à l'aide de micro-jauges de contraintes, l'effort longitudinal (en compressions et dilatations sucessives) s'exerçant sur les tiges de liaison de la plaque supérieure à chacun des hautparleurs ; mais l'ordre de grandeur attendu pour les contraintes, même aux viscosités importantes (de l'ordre de $0,1 \mathrm{~N}$ pour une centaine de poises) reste du même ordre que la sensibilité minimale des jauges. L'autre possibilité, que nous avons retenue, est de mesurer l'effet de la présence d'un milieu visqueux sur le déplacement de la plaque supérieure en maintenant constante la force exercée sur celleci ; les variations de l'amplitude du déplacement restent faibles (diminution de $2 \mu \mathrm{m}$ sur $20 \mu \mathrm{m}$ pour une viscosité d'environ 100 poises), mais les variations de la phase du déplacement sont beaucoup plus importantes $\left(20^{\circ}\right.$ dans les mêmes conditions) et la mesure de l'angle de déphasage est faite par une méthode zéro à l'aide des détections synchrones, ce qui conduit à une précision bien meilleure que dans le cas de la mesure de l'amplitude.

3.3.1 Principe de la mesure. - L'équation différentielle vérifiée par $x$, abscisse d'un point de la tige se déplaçant dans la direction $X$ s'écrit :

$$
m \mathrm{~d}^{2} x / \mathrm{d} t^{2}+\alpha \mathrm{d} x / \mathrm{d} t+k x=F_{0} \cos \omega t
$$

$m$ : masse de tout l'équipage mobile dans cette direction (bobine du haut-parleur, tige, porte-fenêtre et lame supérieure), $\alpha$ : coefficient de frottement fluide global (amortissement fluide dû à la fois au mouvement de la membrane du haut-parleur et à la présence d'un échantillon entre les plaques), $k$ : constante de rappel élastique du haut-parleur, et $F_{0}$ : amplitude de la force magnétique s'exerçant sur la bobine du haut-parleur; nous négligeons dans l'équation ci-dessus la force de rappel due à la flexion du bras perpendiculaire, ce qui est justifié grâce à la présence des « charnières » disposées sur chacun des bras et mentionnées au paragraphe 2.1 ; nous supposons également que la présence d'un fluide entre les plaques n'introduit qu'un terme purement visqueux (proportionnel à la vitesse) et pas de terme élastique (proportionnel au déplacement) ; ceci est justifiable par le fait que le terme d'élasticité qui interviendrait pour un fluide viscoélastique, reste toujours modéré devant le terme de rappel élastique du haut-parleur, aux fréquences et avec les fluides utilisés : la vérification de cette hypothèse sera donnée avec les résultats expérimentaux ( $\$ 3.3 .3$ ci-dessous). 
La résolution de l'équation (1) conduit à :

$$
x=X_{0} \cos (\omega t-\varphi)
$$

avec :

$$
X_{0}=F_{0} /\left\{\left(k-m \omega^{2}\right)^{2}+\alpha^{2} \omega^{2}\right\}^{1 / 2}
$$

et :

$$
\begin{aligned}
\tan \varphi=(\alpha \omega) /( & \left.k-m \omega^{2}\right)= \\
= & (-\alpha / m \omega) /\left(1-\omega_{0}^{2} / \omega^{2}\right)
\end{aligned}
$$

avec $\omega_{0}=(k / m)^{1 / 2}$; dans notre cas, la fréquence de résonance de l'équipage mobile $f_{0}=\omega_{0} / 2 \pi$ (de l'ordre de $45 \mathrm{~Hz}$ ) est suffisamment modérée devant les fréquences utilisées (environ $200 \mathrm{~Hz}$ ), pour que nous puissions écrire :

$$
\tan \varphi \sim(-\alpha) / m \omega
$$

ce qui nous permet de nous affranchir d'une éventuelle variation de la constante élastique $k$ du système (dans le cas de mesures avec un fluide viscoélastique), en supposant bien sûr que l'hypothèse ci-dessus $\left(f_{0} \ll f\right)$ reste vérifiée. Nous disposons donc d'un moyen de mesurer le coefficient de frottement $\alpha$.

Le champ de vitesse $\mathbf{v}(\mathbf{r}, t)$ à l'intérieur de la cellule vérifie l'équation de Stokes :

$$
\partial \mathrm{v} / \partial t=(\eta / \rho) \partial^{2} \mathbf{v} / \partial z^{2}
$$

écrite en négligeant les effets de bords à la périphérie de la cellule. En régime permanent, et lorsque la fréquence d'oscillation de la plaque supérieure est "assez faible », la solution "forcée » de l'équation (4) correspond à une variation linéaire de la vitesse à l'intérieur de la cellule avec la distance $z$ à la plaque inférieure et vérifie :

$$
\begin{aligned}
& \mathbf{V}(Z, t)=\left[V_{0 x} \cos (\omega t+\varphi) \mathbf{u}_{x}+\right. \\
& \left.+V_{0 y} \sin (\omega t+\varphi) \mathbf{u}_{y}\right](z / d)=(z / d) \mathbf{v}(t)
\end{aligned}
$$

où $\mathbf{v}(t)$ est la vitesse (oscillante) de la plaque supérieure, $z$ la côte d'un point à l'intérieur de la cellule, et $\mathbf{u}_{x}$ et $\mathbf{u}_{y}$ les vecteurs unitaires dans chaque direction (Fig. 5). Précisons l'hypothèse « fréquence assez faible »: il faut en fait que la profondeur de pénétration $\delta$ de la perturbation de vitesse créé au niveau de la plaque supérieure $(\delta=(2 \eta / \rho \omega))^{1 / 2}$ soit grande devant l'épaisseur $d$ de la cellule; on peut dire aussi de manière équivalente, qu'il faut que le temps de diffusion de la perturbation de vitesse sur l'épaisseur de la cellule $\left(\sim d^{2} /(\eta / \rho)\right)$ soit petit devant la période d'oscillation de la plaque, ce qui conduit à la même limite; dans notre cas, $\delta \sim 1 \mathrm{~mm}$ pour les plus faibles viscosités $(\eta=10$ Po $)$ et $d \sim 200 \mu \mathrm{m}$. A titre de vérification, nous avons tracé la variation théorique du rapport (amplitude de $\left.V_{x}\right) / V_{0 x}$ en fonction de $(z / d)$ pour

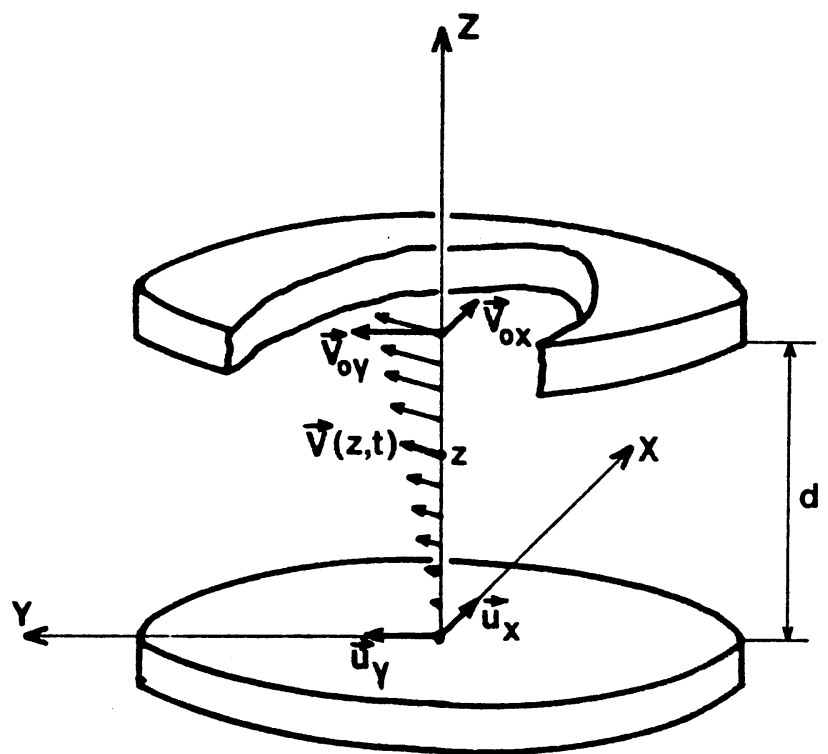

Fig. 5. - Profil de vitesse à l'intérieur de la cellule de cisaillement.

[Velocity profile in the shear cell.]

$\delta / d=1$ et $\delta / d=5$ en repartant de la relation générale pour $V_{x}(z)$ [7] (Fig. 6) ; on constate que le profil de vitesse est tout à fait linéaire dès que $\delta / d>5$.

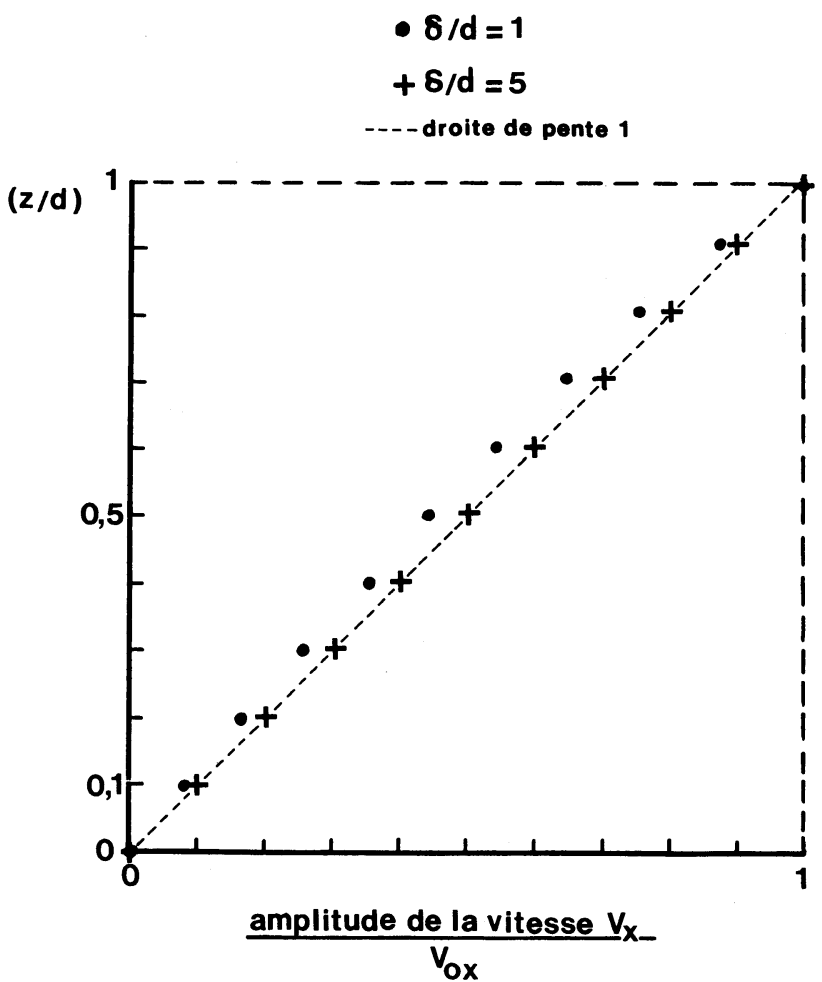

Fig. 6. - Variations de l'amplitude de la vitesse $V_{x}$ en fonction de la cote $z$ à l'intérieur de la cellule, pour deux valeurs de la profondeur $\delta$ de pénétration de la vitesse.

[Normalized amplitude of the velocity $V_{x}$ as a function of the $z$-coordinate, for two values of the viscous penetration depth $\delta]$. 
L'équation (5) nous permet de déterminer les composantes de la contrainte de viscosité exercée sur la plaque supérieure :

$$
\sigma_{z x}=\eta\left[\partial V_{x} / \partial Z\right]_{z=d}=\eta\left(V_{x}(t)\right) / d
$$

et

$$
\sigma_{z y}=\eta\left(V_{y}(t) / d\right)
$$

La force totale correspondante a donc pour composantes :

$$
f_{x}=S \sigma_{z x}=(\eta S / d) v_{x}(t)
$$

( $S$ : surface en contact avec le fluide) et

$$
f_{y}=S \sigma_{z y}=(\eta S / d) v_{y}(t) .
$$

En l'absence de fluide entre les plaques, il existe une force de frottement $\mathbf{f}_{0}=-\alpha_{0} v$. La force de frottement fluide totale est donc:

$$
-\alpha v=-\left[\alpha_{0} v+(\eta S / d) v\right] .
$$

En reportant cette expression dans la relation (3), nous obtenons l'expression :

$$
\tan \varphi=-\left(\alpha_{0} / m \omega\right)-(S / m \omega d) \eta
$$

qui relie la viscosité $\boldsymbol{\eta}$ de l'échantillon, à l'angle de déphasage $\varphi$ entre le courant circulant dans les hautparleurs et le déplacement de la plaque dans chaque direction.

3.3.2 Mode opératoire. - Le déphasage $\varphi_{1}^{\prime}$ entre le courant électrique circulant dans le haut-parleur imposant le déplacement dans la direction $X$, et le signal délivré par la photodiode correspondante est mesuré par une détection synchrone.

Le signal délivré par les photodiodes est en opposition de phase avec les variations d'éclairement ; le déphasage $\varphi_{1}^{\prime}$ entre le courant dans le hautparleur et le déplacement correspondant de la plaque est donc égal à $\left(\pi-\varphi_{1}^{\prime}\right)$.

Le signal donné par l'autre photodiode (mouvement dans la direction $Y$ ) est utilisé de la même manière pour mesurer le déphasage $\varphi_{2}$ entre le courant dans le haut-parleur imposant le mouvement dans cette direction et le déplacement correspondant ; le déphasage $\varphi$ entre les signaux alimentant les haut-parleurs est alors ajusté à une valeur telle que les deux déplacements suivant $X$ et $Y$ soient exactement en quadrature, c'est-à-dire que l'on ait :

$$
\varphi+\varphi_{2}=90^{\circ}+\varphi_{1}
$$

(une représentation des différents signaux sous forme de vecteurs de Fresnel est donnée Fig. 7) ; en fait, les différences entre les déphasages $\varphi_{1}$ et $\varphi_{2}$ sont toujours très modérées (moins de $1^{\circ}$ ) et proviennent d'une légère différence dans les frottements sur les deux voies $X$ et $Y$. Dans le cas de

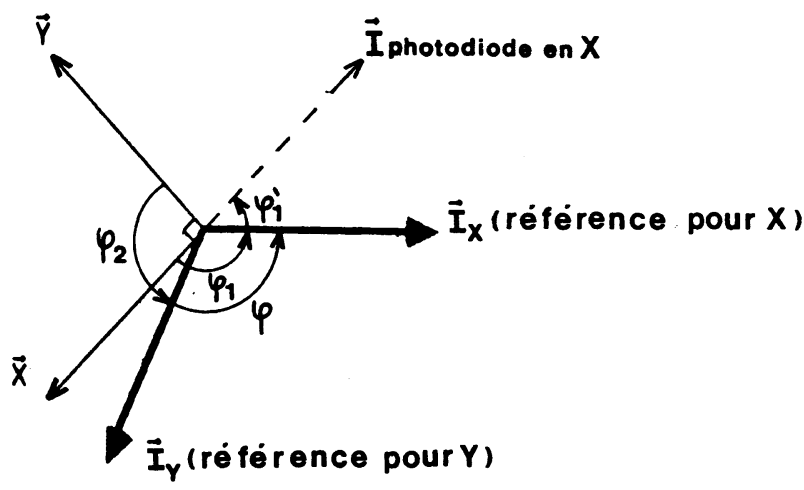

Fig. 7. - Représentation des différents signaux et de leurs phases respectives. $l_{x}$ (resp. $l_{y}$ ) : courant dans le hautparleur créant le mouvement dans la direction $X$ (resp. $Y$ ). $\mathbf{X}$ (resp. Y) : déplacement de la plaque mobile dans la direction $X$ (resp. $Y$ ).]

[Schematic representation of the different signals and their respective phases, $\mathbf{l}_{x}$ (or $\mathbf{l}_{y}$ ): electrical current in the loudspeaker resulting in motion in the $X$ (or $Y$ ) direction, $\mathbf{X}, \mathbf{Y}$ : orthogonal displacements of the moving plate.]

l'étude des suspensions, cet ajustage n'est pas effectué car la différence entre les deux déphasages peut provenir d'un effet d'anisotropie dans la viscosité de l'échantillon (le déphasage $\varphi$ est gardé à la valeur trouvée par ajustement en l'absence de fluide).

3.3.3 Etalonnage et résultats. - Pour vérifier la relation affine entre la viscosité $\eta$ et la tangente de l'angle de déphasage $\varphi_{1}$ (relation (6)), et déterminer en même temps les constantes $\left(\alpha_{0} / m \omega\right)$ et $(S / m \omega d)$ (quantités qu'on ne peut qu'évaluer), nous avons étalonné l'appareil à l'aide d'huiles Silicone (Rhodorsil de la série 47, Rhône-Poulenc) de viscosités variées : nous disposions [8] des viscosités suivantes : 10,50 et 100 poises et avons obtenu des valeurs intermédiaires par mélange des huiles de base (une abaque permet d'estimer la viscosité en fonction de la composition du mélange). Les variations de $\left(-\tan \varphi_{1}\right)$ avec $\eta$ sont représentées sur la figure 8 , la droite tracée correspondant à une régression linéaire. La mesure de la viscosité d'un échantillon se fait par lecture directe sur le graphique, sous réserve que l'épaisseur de l'échantillon soit la même que lors de l'étalonnage $(d=200 \mu \mathrm{m})$; dans le cas contraire une correction est nécessaire pour tenir compte du facteur (1/d) dans le coefficient de $\eta$ de la relation (6) (l'utilisation d'une épaisseur $d$ supérieure à $200 \mu \mathrm{m}$ peut s'avérer nécessaire pour effectuer des mesures avec de plus faibles gradients de vitesses pour étudier le caractère non newtonien des fluides par exemple).

Pour vérifier que les huiles utilisées pour l'étalonnage n'introduisaient qu'un terme d'élasticité négligeable devant celle du système oscillant, nous avons mesuré la fréquence $f_{0}$ pour laquelle le déphasage 


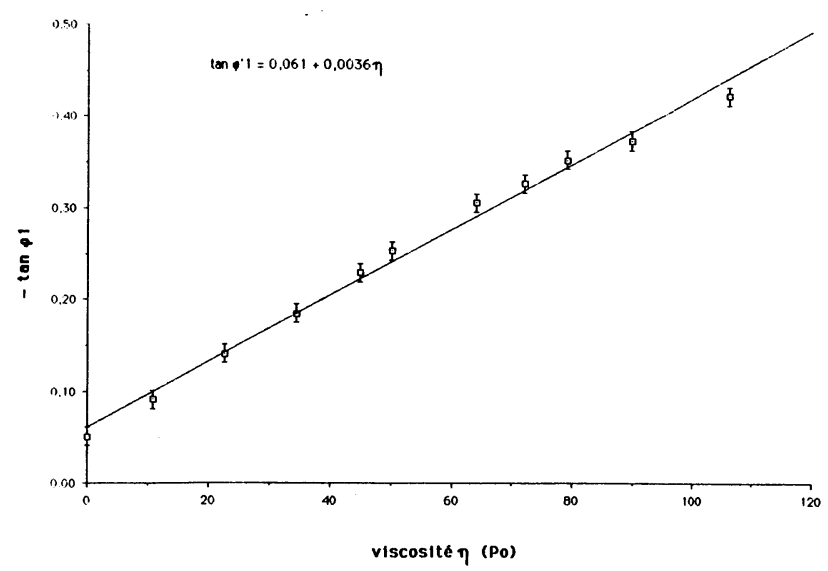

Fig. 8. - Courbe d'étalonnage en viscosité ; la droite tracée correspond à une régression linéaire à partir des points expérimentaux.

[Viscosity calibration curve ; the straight line corresponds to a least-squares fit.]

$\varphi_{1}$ passe par la valeur $90^{\circ}$; en effet, cette fréquence s'écrit :

$$
f_{0}=(k / m)^{1 / 2}
$$

et son changement en présence d'un échantillon est un test de la variation du coefficient d'élasticité $k$ du système ; elle vaut $45 \mathrm{~Hz}$ en l'absence d'échantillon entre les plaques et $47 \mathrm{~Hz}$ (respectivement $49 \mathrm{~Hz}$ ) avec une huile Silicone de viscosité 50 poises (respectivement 100 poises), ce qui nous permet de conclure que l'effet de viscoélasticité du fluide reste négligeable sur le repérage des viscosités.

\section{Conclusion.}

Le système décrit dans le présent article nous permet donc de soumettre un échantillon de fluide en faible quantité (fraction de $\mathrm{cm}^{3}$ ) à un mouvement de cisaillement alternatif polarisé circulairement, elliptiquement ou rectilignement, afin d'étudier les structures induites par le cisaillement ou le mouvement des particules en suspension dans le fluide. Simultanément, nous pouvons évaluer la viscosité du fluide dans la gamme allant de quelques dizaines à quelques milliers de poises.

Nous poursuivons actuellement ce type d'expérience sur des suspensions constituées de billes de verre (diamètre $40 \mu \mathrm{m}$ ) placées dans une huile assez visqueuse (50 poises) pour différentes concentrations de billes [6].

Ce travail bénéficie d'un soutien du C.N.R.S. dans le cadre d'une Action Thématique Programmée sur l'étude des milieux aléatoires macroscopiques.

\section{Bibliographie}

[1] Prost, J., Dubois-Violette, E., Guazzelli, E., Clement, M., Smectics : A Model for Dynamical Systems?, Cellular Structures in Instabilities, Lectures Notes in Physics 210 (1984) 215 (Springer Verlag).

GuAZZELli, E., KoCH, A., Hydrodynamic Instabilities in a periodically sheared Nematic Liquid Crystal, J. Phys. 46 (1981) 673.

La cellule de cisaillement décrite dans le présent article et les références ci-dessus est dérivée d'un premier dispositif expérimental mis au point par Dreyfus, Guyon et Pieranski [3, 4] dans lequel chacun des deux plans parallèles entre lesquels est inséré le fluide est animé d'un mouvement oscillatoire dans une direction $(X$ pour le plan supérieur, $Y$ pour le plan inférieur); la deuxième cellule décrite ici permet d'avoir une ellipticité du cisaillement (rapport des amplitudes de déplacement dans chaque direction) constante sur toute l'épaisseur de l'échantillon.

[2] Dubois-Violette, E., Rothen, F., Instability of a homeotropic nematic subjected to an elleptical shear : theory, J. Physique 39 (1978) 1039.

[3] Dreyfus, J. M., GuYon, E., Convective instabilities in nematics caused by an elliptical shear, $J$. Physique 42 (1981) 283.
[4] Dreyfus, J. M., Pieranki, P., Distorsion Waves and phase slippage in nematics, J. Physique 42 (1981) 459.

[5] Gauthier Manuel, B., Meyer, R. and Pieranski, P., The sphere rheometer, J. Physique E 17 (1984) 1177.

[6] Noetinger, B., Petit, L., Mise en évidence expérimentale de structures induites sur des suspensions concentrées soumises à un cisaillement alternatif, C.R. Hebd. Séan. Acad. Sci. 303 sér. II (1986) 1269.

Nous remercions P. Gentic pour sa collaboration au démarrage de cette étude.

[7] L'expression générale de l'amplitude $Y_{0}(z)$ de la vitesse $Y(z, t)$ est :

$V_{0}(z)=V_{0}\left[\frac{\cosh (2 z / \delta)-\cos (2 z / \delta)}{\cosh (2 d / \delta)-\cos (2 d / \delta)}\right]$

où $V_{0}$ est l'amplitude de la vitesse en $z=d$ (plaque supérieure) (voir par exemple Jaeger et Carslaw conduction of heat in solids (Oxford Press) 1959, p. 105).

[8] Nous remercions la société Rhône-Poulenc pour nous avoir fourni gracieusement des échantillons de certaines viscosités. 